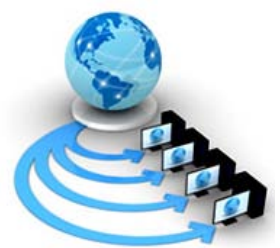

Volume 8, No. 7, July - August 2017

International Journal of Advanced Research in Computer Science

REVIEW ARTICLE

\author{
Available Online at www.ijarcs.info
}

\title{
A SURVEY ON BRAIN TUMOR IDENTIFICATION THROUGH MEDICAL IMAGES
}

\author{
Mehak Saini \\ Deenbandhu Chhoturam University \\ of Science and Technology Murthal, \\ Sonepat, Haryana, India
}

\author{
Priyanshu Tripathi \\ Hindu College of Engineering \\ Sonepat, Haryana, India
}

Madhwendra Nath

\author{
Hindu College of Engineering \\ Sonepat, Haryana, India
}
Sanju Saini
Deenbandhu Chhoturam University of Science and
Technology Murthal, Sonepat, Haryana, India

\author{
K K Saini \\ Hindu College of Engineering \\ Sonepat, Haryana, India
}

\begin{abstract}
This paper presents a survey on the detection of brain tumor. Brain Tumor can be described as a cluster of abnormal cells which grows inside the brain by uncontrolled growth in tissues of the brain which needs to be treated, If left untreated it can grow beyond to other parts of brain too. It is very difficult and challenging to detect the brain tumor in initial phase as there are very few or no symptoms in the early stage. Detecting the brain tumor in early stage can provide more treatment options. There are various methods which can be used to detect the tumor such as Segmentation, Thresholding, Fuzzy clustering or Artificial neural network.
\end{abstract}

Keywords: Brain Tumor, Magnetic Resonance Imaging (MRI), Image Segmentation, Thresholding, ANNs, FCM.

\section{INTRODUCTION}

Brain Tumor is a disease of abnormal cells growing and multiplying in an undisciplined manner. It has become one of the most common and major causes for the increase in Mortality among children and adults in the world. Brain tumor cells grow inside and along the brain. Brain is portion of central nervous system which is located into the skull. It is soft spongy mass of tissue that is protected by bones of skull and three slim membranes called meninges.

Brain tumor is cluster of abnormal cells which indirectly destroys healthy cells of brain and causes inflammation, brain swelling and pressure within the skull. Brain tumors can be divided into main two types one is benign and second one is malign. Brain tumor can be found in different sizes and shapes which differ case by case. But the effects of both the types of tumor are mostly same and can cause similar problems depending on their kind. Benign Tumor is a non cancerous type of tumor which does not grow in nearby tissues or spread to other parts [1].

Malignant tumors are cancerous tumor and are dangerous since they spread to distinct parts of body. Tumors in critical area of brain can cause weakness, stiff muscles or problem with sensation, facial or eye movement such as Double vision is a common early symptom of brain stem tumors. The grade of tumor refers to way the cells look under a microscope.

$>$ Grade 1- Benign tissues, these tissues looks like normal tissues slowly give rise to these cells.

$>$ Grade 2- The cells are malignant these tissues looks like normal tissues alike grade- 1 tissues.

> Grade 3- They are the malignant tissues which are different from normal cells. As they are abnormal cells so are fast growing.
Grade 4- These are mostly abnormal cells and are rapidly spread.

In this paper, the reviews of techniques have been done which can be used to detect the tumor such as Segmentation technique, Thresholding technique, Edge detection, Watershed segmentation etc. Brain tumor segmentation and detection using Magnetic Resonance Imaging has become as one of the most popular research field in medical imaging system during the past some years [2].

This paper reviews the proposed strategies in identification of the brain tumor from MRI as well as CT scan images of the brain.

\section{RELATED WORK}

In 2009, Ahmed Kharrat et al.[3] proposed the methodology of enhancement, segmentation and classification for detection of brain tumor. In Enhancement, it is used in medical imaging to make images clearer and ensure optimum presentation. It aims at improving the quality of images. After Preprocessing, Segmentation algorithm is used. The main aim of segmentation is dividing or portioning the image into homogeneous regions. After this, classification is used to regroup heterogeneous elements into a small no. of classes.

In 2010, T.Logeswari et al[4] proposed the segmentation methods into two phases. In first phase, the MRI image of brain is acquired from the data and noises are removed from it. In second phase, Hierarchical Self Organizing Map(HSOM) is employed for image segmentation. A Self Organizing Map(SOM) is a type of neural network. SOM works in two stages: Training and mapping. Where training is a competitive process and Mapping automatically Differentiates a new input vector. In a CAD analysis 
execution time is an important parameter of image segmentation.

In 2011, K.S. Angel Viji et al.[5] proposed that after a manual segmentation procedure of the tumor identification, the investigations has been done for the potential use of MRI data for enhancing brain tumor shape approximation and also for 2D \& 3D visualization for surgical planning and assessing tumor. The CAD system provides the precious outlook and accuracy of earlier brain tumor detection. The steps followed are image acquisition then preprocessing \& enhancement and last is feature extraction \& selection.

In 2011, Mohammed J. Islam[6] discovered that computer vision is the best solution for real time checking of capsules and medicines in pharmaceutical industries in which the author has made a system for inspection of quality using edge based segmentation technique.

In 2012, Nagalkar et al. reviews a work on CT scan technique which is used for monitoring the images of part of the brain which is damaged. The RGB components are invisible in CT Scan images. In CT scan images the tumor is shown white in color and the damaged cells of brain are shown in black color, therefore the values of pixels shows the destroyed brain cell as 0 and the tumor cells as 1 . Thus the extraction of the tumor can plotted in MATLAB [7].

In 2012, Pratibha Sharma et al[8], proposed an efficient algorithm for the detection of edges of brain tumor. In which the initial stage is image acquisition of MRI scan then the noise filters are used to remove the noises from the MRI Scan images then the images are enhanced and at last the morphological operations are used for the extraction of region suffering from tumor. Noise removal can be done using filters such as linear \&non linear filters. Using these methods gives accurate and clear results.

In 2012, S. Kobashiet. al.[9] proposed image segmentation using scale based Fuzzy and fuzzy object model to segment the parenchym of part of newly born brain MRI scan image. In first step the suthor has separated foreground region. In next step MRI intensity homogeneity is corrected and lastly the scale base Fuzzy object model is applied to the final images. This method has given maximum accuracy.

In 2014, Muhammad WaseemKhan[10] reviewed the different Image segmentation techniques such as Threshold, edge detection and Region based. In this paper he has reviewed techniques which are Edge based detection, Fuzzy theory based segmentation, PDF based segmentation, ANN (Artificial Neural Network) based segmentation, Threshold segmentation, Region based segmentation.

In 2015, S. Mahalakshmiet. al.[11], proposed a detection and separation of brain tumor through MRI images using Particle Swarm Optimization(PSO) which is a heuristic global optimization method on swarm intelligence. It works in four steps: Conversion then Implementation , Selection and finally Extraction [12]-[17].

\section{DISCUSSION}

In this paper, a review is done on different data mining and image processing methods or techniques which can be used in automated brain tumor detection system. In this paper, various techniques for brain tumor detection have been reviewing which may be employed in recent years for the detection of brain tumor but the main focus was on different image segmentation techniques for the detection of brain tumor. For Brain tumor detection MRI images are widely used however CT scan image are widely used for Lung cancer detection [18]-[21]. The summary of different segmentation classification based on the years they were used and their algorithm has been presented in Table 1.

TABLE 1: Summary of Various Techniques for Brain Tumor Detection

\begin{tabular}{|l|l|l|l|}
\hline Author & Images & $\begin{array}{l}\text { Different Method used } \\
\text { for Image Segmentation }\end{array}$ & Year \\
\hline $\begin{array}{l}\text { Ahmed } \\
\text { Kharrat }\end{array}$ & $\begin{array}{l}\text { MRI \& } \\
\text { CT Image Enhancement }\end{array}$ & 2009 \\
\hline T.Logeswari & MRI & Soft Computing & 2010 \\
\hline $\begin{array}{l}\text { K.S. Angel } \\
\text { Viji }\end{array}$ & MRI & CAD System & 2011 \\
\hline $\begin{array}{l}\text { Nagalakr V.J } \\
\text { CT }\end{array}$ & Soft Computing & 2012 \\
\hline $\begin{array}{l}\text { Pratibha } \\
\text { Sharma }\end{array}$ & MRI & Edge Detection & 2012 \\
\hline $\begin{array}{l}\text { S.Mahalaksh } \\
\text { mi }\end{array}$ & MRI & $\begin{array}{l}\text { Particle } \\
\text { Optimization }\end{array}$ \\
\hline
\end{tabular}

\section{REFERENCES}

[1.] Dr.M.Karnan, T. Longeshwari, “Improved Implementation of Brain MRI image Segmentation using Ant Colony System”, IEEE 2010, 978-1-4244-5967-4/10.

[2.] Selvanayaki, K., \&Karnan, M. (2010). CAD system for automatic detection of brain tumor through magnetic resonance image-a review. International Journal of Engineering Science and Technology, 2(10), 5890-5901.

[3.] Ahmed Kharrat et al.,"'Detection of Brain Tumor in Medical Images”, 2009 International Conference on Signals, Circuits and Systems.

[4.] T.Logeswari, M.KARNAN,”An Enhanced Implementation of Brain Tumor Detection Using Segmentation Based on Soft Computing”, 2010 International Conference on Signal Acquisition and Processing.

[5.] K..S. Angel Viji et al,"Automatic Detection of Brain Tumor based on Magnetic Resonance Image using CAD System with watershed segmentation”, Proceedings of 2011 International Conference on Signal Processing, Communication, Computing and Networking Technologies (ICSCCN 2011).

[6.] M. J. Islam, S. Basalamah, M. Ahmadi, and M. A. S. hmed, "Capsule image segmentation in pharmaceutical applications using edge-based techniques," IEEE International Conference on Electro/Information Technology (EIT), pp. 15, 2011.

[7.] NAGALKAR V.J. AND ASOLE S.S., "Brain Tumor Detection Using Digital Image Processing Based On Soft Computing”, Journal of Signal and Image Processing ISSN: 0976-8882 \& E-ISSN: 0976-8890, Volume 3, Issue 3, 2012, pp.-102-105.

[8.] Pratibha Sharma et al,"Application of Edge Detection for Brain Tumor Detection”,International Journal of Computer 
Applications (0975 - 8887) Volume 58- No.16, November 2012.

[9.] S. Kobashi and J. K. Udupa, "Fuzzy object model based fuzzy connectedness image segmentation of newborn brain MR images," in Proc. IEEE International Conference on Systems, Man, and Cybernetics (SMC), pp. 1422-1427, 2012.

[10.] Muhammad Waseem Khan,“A Survey: Image Segmentation Techniques”,International Journal of Future Computer and Communication, Vol. 3, No. 2, April 2014.

[11.]S. Mahalakshmi* and T. Velmurugan,"Detection of Brain Tumor by Particle Swarm Optimization using Image Segmentation”, Indian Journal of Science and Technology, Vol8(22), DOI:10.17485/ijst/2015/v8i22/79092 September 2015.

[12.] Sanju Saini, Ketan Kumat et al., "Solar Tracking: An efficient method of improving solar plant efficiency", International Journal of Electrical and Electronics Engineers, Vol. 7, Issue 1, June 2015.

[13.]Sanju Saini, Sarita Rani, "Temperature control using intelligent techniques", IEEE conference on advanced computing and communication technologies", 2012, pp. 138145.

[14.]V. Rohilla, K.P.S. Parmar, S. Saini, "Optimization of AGC parameters in the restructured power system environment using GA", International Journal of Engineering Science and Emerging Technologies, Vol. 3, No. 2, pp. 30-40, Oct. 2012.

[15.]Rajbala, Mrs. Sanju Saini, “ MATLAB Implementation of Memristor based Chua's Circuit and its Chaos Control”, Proc. of Int. Conf. on Emerging Trends in Engineering and Technology, DOI: 03.AETS.2013.3.279 (C) Association of Computer Electronics and Electrical Engineers, 2013

[16.]Priyanshu Tripathi et.al.,"Occupancy Grid Mapping for Mobile Robot using Sensor Fusion,”Proc. IEEE Int'l Conf. on Issues and Challenges in Intelligent Computing Techniques (ICICT), KIET GHZ ,INDIA, pp. 49-53, Feb. 2014.

[17.]Madhwendra Nath et al.,"Identification of First and Second Heart Sound using Shannon Energy of Heart Sound Signal” Int'l Conf. in CGI, Fatehgarh Sahib, Punjab, Feb 2010.

[18.]Mehak Saini, Priyanshu Tripathi et. al., "A Review on Lung Cancer Segmentation Techniques,” International Journal of Engineering Science and Technology, Vol. 9, Issue. 3, pp. 190-194, March 2017.

[19.]Mehak Saini, Priyanshu Tripathi et. al., "Digital Image Watermarking Techniques And Attacks: A Review,” International Journal of Engineering Science and Technology, Vol. 9, Issue. 4, pp. 240-244, April 2017.

[20.] Mehak Saini, Madhwendra Nath, Priyanshu Tripathi et. al., "Computation and Analysis of Heart sound signal using Hilbert Transform and Hilbert-Huang Transform,"International Journal of Engineering and Technology, Vol. 9, Issue. 2, pp. 1462-1468-194, Feb. 2017.

[21.]Mehak Saini,Madhwendra Nath, Priyanshu Tripathi et. al., "Investigatory Analysis of Attacks on DSR and AODV in Mobile Ad-hoc Network," International Journal on Computer Science and Engineering, Vol. 9, Issue. 4, pp. 122-128, April, 2017.

\section{BIOGRAPHIES}

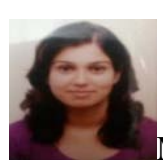

Ms. Mehak Saini is B.Tech (ECE) and pursuing M.Tech from Deenbandhu Chhoturam University of Science and Technology Murthal, Sonepat ,Haryana, India. She is a young Technocrat and Researcher. She has published 6 research papers in National/ International Journals. Her area of Interest is Watermarking Techniques, Optical Communication and Advanced Communication System.

Mr. Priyanshu Tripathi is B.Tech., M.Tech from N.I.T. Jalandhar, Punjab, India. He is a young Technocrat and researcher. Currently, $\mathrm{He}$ is working as an Assistant Professor in Hindu college of Engineering Sonepat, Haryana, India. His area of Interest is Robotics and image processing. He has published various research papers in International journals and IEEE international conferences.

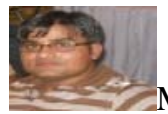

Mr. Madhwendra Nath is B.Tech, M.Tech from N.I.T. Jalandhar, Punjab, India. Currently, He is working as an Assistant Professor in Hindu college of Engineering Sonepat, Haryana, India. His area of Interest is Signal Processing and image Biometric Security. He has published various research papers in National, International and IEEE international conferences.

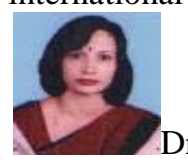

Dr. Sanju Saini is B.Tech.., M.Tech. \& PhD in Electrical Engineering. Currently, She is Assistant Professor in Deenbandhu Chhoturam University of Science and Technology Murthal, Sonepat ,Haryana, India. His area of interest is Control Syatem, Chaos based Nonlinear Dynamic System and Artificial Neural Networks. She has published more than 30 research papers in various reputed National and International journals and conferences. She has guided Dissertation of more than 20 M.Tech. Students.

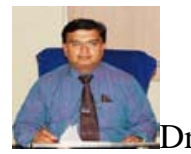
$\mathrm{PhD}$ in Electronics \& Communication Engineering. Currently, He is Director-Principal of Hindu College of Engineering Sonepat, Haryana, India. His area of interest is Optical Communication, Chaos Communication, Satellite Communication and Reliability Engineering. He has published more than 500 research papers in various reputed National and International journals and conferences. He has guided Dissertation of more than 100 M.Tech. students and 7 Ph.D. scholars. For more detail kindly visit the website www.drkksaini.com. 